\title{
Pulsating Cool Stars and Galactic Structure
}

\author{
Patricia Whitelock \\ SAAO, P O Box 9, Observatory, 7935, Western Cape, South Africa. \\ (e-mail:paw@saao.ac.za)
}

\begin{abstract}
The review concerns the use of Miras and OH/IR stars as probes of Galactic structure and concentrates on groups of stars with both kinematic and luminosity data. The reliability of distance determinations for these stars is covered in some depth. Kinematic and scale height data for local Miras suggest that those with pulsation periods of around 200 day are members of the thick disk or inner halo. Longer-period stars are kinematically cooler and have smaller scale heights. Stars with moderate dust shells, which may be more evolved, seem to belong to the same population as thin-shelled sources with the same pulsation period. Our current knowledge of the structure of the Galactic Bulge is reviewed, as is our understanding of its rotation as derived from observations of $\mathrm{SiO}$ Masers from Mira variables. Infrared and radio surveys, in progress or planned, offer us the potential to extend the study of low-mass metal-rich stars well beyond the solar neighbourhood.
\end{abstract}

\section{Introduction}

This paper concentrates on the use of Miras and their long-period analogues, the $\mathrm{OH} / \mathrm{IR}$ stars, (henceforth collectively referred to as Miras) as probes of Galactic structure. Other aspects of these stars, their evolutionary status, their pulsation mode(s), etc., are dealt with in other papers in this volume, in particular those by Chapman, Wood and Tuchman.

I want to start by looking at why we might be interested in using Miras as probes of Galactic structure, and at the advantages they have over more traditional probes. First, we know from observations of Miras in globular clusters and from the narrowness of the period-luminosity (PL) relation, that stars of a particular age or initial mass will become Miras with a particular period and that there can be rather little evolution of that period with time. Therefore, by choosing Miras within a particular period range, we can isolate a particular population. Secondly, the Mira evolutionary phase is the most luminous one that any given star ever reaches. For low-mass stars a Mira will be several thousand times brighter than its main sequence progenitor. Thus, in principle, the star can be observed at far greater distances than it can at other stages of its evolution. Thirdly, the Mira phase is short lived so every Mira represents at least $10^{4} M_{\odot}$ of stars, with the same age, metallicity, similar mass etc. Fourthly, their circumstellar environments produce Maser emission which can be detected over very large distances unhindered by interstellar extinction. These Masers 
have enabled kinematic studies to be made of regions largely inaccessible by other techniques, in particular near the Galactic Centre.

There are of course a few difficulties associated with the use of these AGB stars as Galactic structure probes. Some of these are fundamental and others merely a function of our current ignorance. In the latter category, the most serious is our rather poor understanding of the pulsation and evolution of these stars; there are no satisfactory models for their atmospheres nor unanimity on their pulsation mode; neither do we understand how to integrate mass-loss, the dominant evolutionary process, into the evolutionary theory. This lack of understanding has led to obvious questions about the applicability of the PL relation, particularly for stars with periods in excess of about 420 day (see \$2). The short lifetimes of the stars are a disadvantage as well as an advantage: we are required to examine large volumes in order to find statistically significant samples. Perhaps the biggest disadvantage is the time-consuming nature of the observations: typically a dozen measurements spaced over several years are required to establish the approximate period of one of these stars. The bolometric amplitudes are large, over a magnitude for long-period stars, so a single measurement is not necessarily a good approximation to the mean magnitude. Other useful measurements, such as phase-lag distances are extremely time consuming.

\section{Measuring distances}

The following five methods are the most commonly used to determine distances of Miras: (1) Assume they all have the same bolometric or infrared luminosity; (2) A colour-dependent bolometric correction to an adopted luminosity; (3) Kinematic Distance; (4) PL Relation; (5) Phase-lag method for OH/IR stars. Neither the assumption that all AGB variables have the same luminosity nor the use of a colour dependent bolometric corrections works very well unless the sample is carefully selected to have a very narrow range of properties. Kinematic distances are derived assuming the stars follow circular orbits within a model of Galactic rotation. It is clear from the pioneering kinematic studies by Baud et al. (1981) that only the $\mathrm{OH} / \mathrm{IR}$ stars with large $\mathrm{OH}$-expansion velocities follow circular orbits. These stars with large expansion velocities are the most luminous and presumably have the most massive progenitors. Habing (e.g., 1988) has made this particular point at various times but even in the recent literature you will find attempts to disprove the validity of the PL relation via kinematic distances using $\mathrm{OH} / \mathrm{IR}$ stars with only moderate expansion velocities.

The period-luminosity relation is an important method and, judging from the recent literature, a source of considerable confusion. The PL relation for Miras with periods less than 420 day (which constitute about $70 \%$ of the Miras in the solar neighbourhood) was discussed by Whitelock et al. (1994). They showed that a single PL relation is obeyed by Miras in the LMC, the Galactic globular clusters and the solar neighbourhood. Feast (1988) and Peter Wood (1995 private communication) have shown that this same relation is also obeyed by Miras in the SMC. It is clear that there is no justification for making theoretical metallicity corrections to the observed PL relation. Relations for both bolometric and $\mathrm{K}$ magnitude have been derived. Of the two, the bolometric one is to be prefered because the $\mathrm{K}$ relation breaks down for sources with thick dust 
shells when circumstellar extinction becomes important at $2.2 \mu \mathrm{m}$. Nevertheless the $K$ relation does have the advantage that the same relation applies to both oxygen- and carbon-rich Miras.

The LMC contains Miras with periods in excess of 420 day which clearly lie above an extrapolation of the PL relation for shorter period stars (e.g., Feast et al. 1989). At very much longer periods ( $\mathrm{P} \gtrsim 1000$ day) $\mathrm{OH} / \mathrm{IR}$ stars are found in the LMC which again lie on the extrapolation of the PL relation derived from short period Miras (Wood et al. 1992). One might anticipate that stars exist with $420<\mathrm{P}<1000$ day which also obey the PL relation but they will have shells sufficiently thick to make them optically invisible and of too low a luminosity to be detected by IRAS. We expect to find out about them from surveys using the ISO satellite.

Our knowledge of Galactic stars with periods over 420 day is still very limited. Lindqvist et al. (1992) discovered $134 \mathrm{OH} / \mathrm{IR}$ stars within a degree of the Galactic Centre, which we might reasonably assume to be at the distance of the Galactic Centre. Van Langevelde (1992) determined periods (mostly $450<\mathrm{P}<1000$ day) and luminosities for several of these OH/IR stars and finds that on average they fall distinctly below an extrapolation of the PL relation if the distance to the Galactic Centre is $8 \mathrm{kpc}$. Recently Jones et al. (1994) redetermined periods and luminosities for some of these sources, finding somewhat different results and on average a higher luminosity at a given period. My impression is that the measurements are too uncertain to draw definite conclusions on the PL relation, but further observations of these stars are very important and the whole problem is well worth pursuing. It should be made clear that these are very difficult measurements to make; the crowding is horrendous and the corrections for interstellar extinction are large and uncertain.

Ultimately we might hope to get the best distances to OH/IR stars from the phase-lag technique (Chapman 1995) but the published measurements to date are not sufficiently accurate to critically test the PL relation (Whitelock 1990). This situation should change in the near future as results from the Australia Telescope and Hartebeesthoek become available.

To summarise the status of Mira luminosities: the PL relation is well established for Miras with periods less than about 420 day. The absolute luminosities of longer-period stars remains very uncertain. There is also a PL relation for $\mathrm{C}$ Miras in the LMC but we have no real idea how well it applies to Galactic stars. Thus, from the point of view of using Miras as Galactic structure probes among populations of low mass stars, the situation is quite good. Most Miras in the solar neighbourhood have periods less than 420 day. We only know about the very long-period $\mathrm{OH} / \mathrm{IR}$ sources as a result of Galactic Plane $\mathrm{OH}$ surveys to very large distances.

\section{The solar neighbourhood}

Before discussing recent work on Miras in the solar neighbourhood I want to remind you of the main result from Feast's (1963) kinematic analysis. This work demonstrated rather clearly that the asymmetric drift and velocity dispersion of the Miras are functions of their pulsation period and that different period Miras belong to different populations. It is this conclusion from Feast's work which 
has, more than anything else, been responsible for the approach of those of us working from South Africa, and for the importance we have placed on measuring the periods of the Miras we have studied.

Note that Galactic Structure studies are limited by the completeness of surveys for Mira variables. In recent years the compilation of the GCVS has been supplemented by new variables discovered largely via the two-micron sky survey, the AFGL survey, the IRAS survey and various $\mathrm{OH}$ surveys of the Galactic Plane. None of these covered the entire sky, although IRAS was not far off. Follow-up observations are necessary to determine the period of a Mira once its nature has been recognised. The follow-up work, even on the surveys finished some years ago, is incomplete, so we do not yet have a complete sample of Miras with known periods even within one kpc of the Sun. Although it is probably very close to being complete for Miras with periods of over 300 day within one kpc. If the DENIS and 2MASS surveys are followed by systematic efforts to determine periods, etc. the situation will improve dramatically.

I want to concentrate on a few recent studies, in particular those by Jura \& Kleinmann (1992, henceforth JK92), Jura et al. (1993, henceforth JYK93), Jura (1994) and Whitelock et al. (1994). The approach taken by Jura and collaborators in looking at the space distribution of the Miras is a particularly useful one; they divided the Miras into groups according to their periods and used infrared data, rather than the less reliable optical data, to derive distances. They use a modified version of the K PL relation to derive distances. As shown by Whitelock et al. (1994) this modified PL relation is incorrect and in the following discussion all distances are corrected to the PL relation used by Whitelock et al. In addition to discussing separately short-, intermediate- and long-period Miras Jura \& Kleinmann (1989) also isolate a separate group of dust enshrouded $A G B$ stars and examine their properties, in this case without considering their periods. It is possible to trace periods and other data on a large fraction of these stars which shows them to comprise: 25 oxygen- and 16 carbon-rich Miras with $270<\mathrm{P}<1600$ day, 7 oxygen- and 11 carbon-rich Miras with unknown periods and 4 non-Miras. Although this constitutes an interesting group of stars worth further investigation, it is too inhomogeneous a group to be useful for Galactic structure studies.

The stars examined by Whitelock et al. (1994) were colour-selected IRAS sources from the South Galactic Cap. There were 61 stars, 35 of which were already known to be Miras by the GCVS, although not all had known periods. Periods were determined for all of them. The kinematics of this sample were also examined, using velocities from the literature together with new ones determined by John Menzies. Figure 1 shows the radial velocity and the height above the plane as functions of period. The distances were calculated from the PL relation. The open circles are $\mathrm{C}$ stars, all of the others are oxygen-rich Miras. These diagrams qualitatively, at least, reinforce what we saw from Feast's work. The 200 day Miras have a large velocity dispersion and occur at large distances from the plane. Longer period stars belong to kinematically cooler populations and presumably originate from more massive progenitors.

Table 1 lists some standard numbers for different populations for comparison with the Miras: the scale height $\left(\mathrm{Z}_{0}\right)$ above the Galactic plane, the velocity dispersion $\left(\sigma_{Z}\right)$ in the same direction and the asymmetric drift $\left(\mathrm{V}_{\mathrm{A}}\right)$. These 
Table 1. Local Populations (Freeman 1987; Carney 1993)

\begin{tabular}{lccc} 
& $\begin{array}{c}\mathrm{Z}_{0} \\
(\mathrm{pc})\end{array}$ & \multicolumn{2}{c}{$\sigma_{z}$} \\
$\left(\mathrm{kms}^{-1}\right)$ & $\mathrm{V}_{\mathrm{A}}$ \\
\hline Halo & - & 75 & $\sim 220$ \\
"Thick Disk" (IP II) & 1000 & 40 & 30 to 40 \\
Old Disk & 350 & 20 & 15
\end{tabular}

values are typical of those quoted by many people over the last few years. Table 2 lists the same quantities for Miras in the various period groups. The kinematic information is from Feast et al. (1972) and the scale heights are from JK92/JYK93. Although the samples are not drawn from the same volume, there is overlap between the stars from Feast and those from Jura. The scale heights were obtained via the median height above the plane. As incompleteness will be most serious among distant stars the scale heights are treated as lower limits. The shortest period group $(P<145$ day $)$ do not fit the pattern of the other stars; they are something of an enigma and there is no room to discuss them here. The 150-250 day Miras have kinematics which are intermediate between the halo and the so called 'thick disk' (or intermediate population II). This is an interesting observation in itself as current ideas for the formation of the thick disk tend to favour a discontinuity between the disk and halo populations. JK92 derived a single scale height for all stars with periods less than 300 day; however, their sample is dominated by the 200 to 300 day group. For the 145 to 200 day group alone the scale height calculated from the data in JK92 is about 700 pc. On the basis of the kinematics of this period group, particularly the asymmetric drift, we expect these stars to be transition objects between the thick disk and the halo. The scale height is more suggestive of the thick disk. The longer-period stars have much smaller scale heights, as the kinematics suggest they should, and look more like old-disk stars.

Jura (1994) published a list of 14 stars (his table 4) of which he suggested most (about 10) were members of the thick disk ("E/T disk"), the kind of object he suggests the short-period $(\mathrm{P}<300)$ Miras would evolve into towards the end of their Mira phase when they have relatively thick dust shells. These stars were selected from $|b| \geq 30^{\circ}$, with $\mathrm{F}_{\nu}(12 \mu \mathrm{m}) \geq \mathrm{F}_{\nu}(2.2 \mu \mathrm{m})$ and $|\mathrm{Z}| \geq 600 \mathrm{pc}$. Jura calculated the distances assuming a bolometric luminosity of $10^{4} L_{\odot}$. Of the 14 stars listed, one is a semi-regular variable (03287-1535: see Whitelock et al. 1995), one is a carbon star $(02152+2822)$ while the others are oxygen-rich Miras with periods (available for 11 out of 12 of the stars) in the range 425 to 559 day. Their kinematics (see Table 3, from velocities in the literature) suggest an old-disk population, with a scale height between 300 and $400 \mathrm{pc}$. The velocities and distances from the plane of these stars are compared in Fig. 1 with those of the IRAS selected group from Whitelock et al. (1994), also listed in Table 3 (there are 7 stars in common). There are no grounds for associating these stars with the kinematically hotter, shorter-period group. Jura (1994) also suggested that the circumstellar gas outflow velocities for these stars were lower than for typical dusty stars. A comparison with Fig. 1 from Sivagnanam et al. (1989) indicates that the expansion velocities are within the normal range found for stars of the same period. More broadly speaking we see from the scale heights, 
Table 2. Miras (Feast et al. 1972; JK92; JYK93)

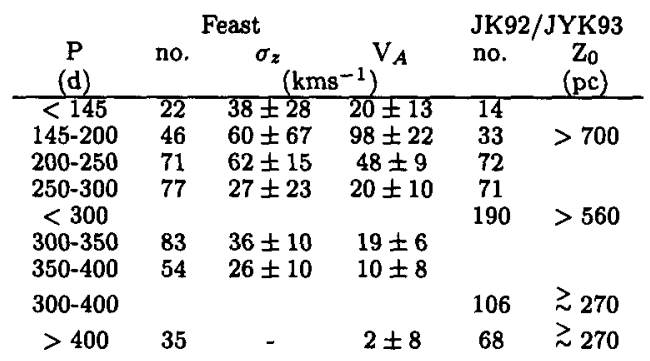

Table 3. IRAS selected stars from $|b|>30^{\circ}$

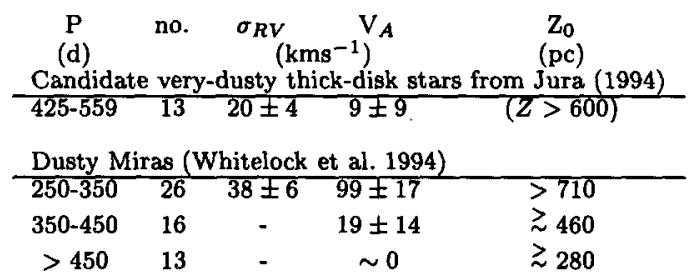

velocity dispersions and asymmetric drifts in Table 2 that the 200 day Miras are plausibly an extreme part of the thick disk and that there is a gradual change of properties as we go to longer periods, until we get to the Miras with periods of around 400 day with properties which suggest an association with the old disk. Presumably the long-period OH/IR stars belong to a still younger population. The one outstanding inconsistency between this picture and the data presented here is the asymmetric-drift of the 250 to 350 day Miras from Table 3; this is similar to the large drift of the much shorter period Miras from Table 2. The velocity dispersion of this group is what we would expect for their periods, but the uncertainties are too large to enable us to draw a firm conclusion from this. There seem to be two possible explanations for this asymmetric drift. Majewski (1993) has summarised evidence suggesting that the asymmetric drift of the thick disk increases with distance from the plane. More recent work (e.g., Ojha et al. 1994) suggests that the gradient in asymmetric drift is small and possibly zero. If there is a gradient in the asymmetric drift then it might explain the difference between the drift of the Miras from the solar neighbourhood (Table 2) and those from the South Galactic Cap (Table 3) as the latter sample contains a large fraction at considerable distances from the plane. This point is obviously critical to the interpretation of these kinematics. An alternative explanation is that these dusty 250-350 day Miras have evolved from the shorter period stars without dust shells. If this were the case we would have overestimated their distances with the PL relation and the scale height would be smaller. There are two crucial measurements which would clarify this point. One is good distances to a number of the dusty Miras, and the second is a much more complete survey 

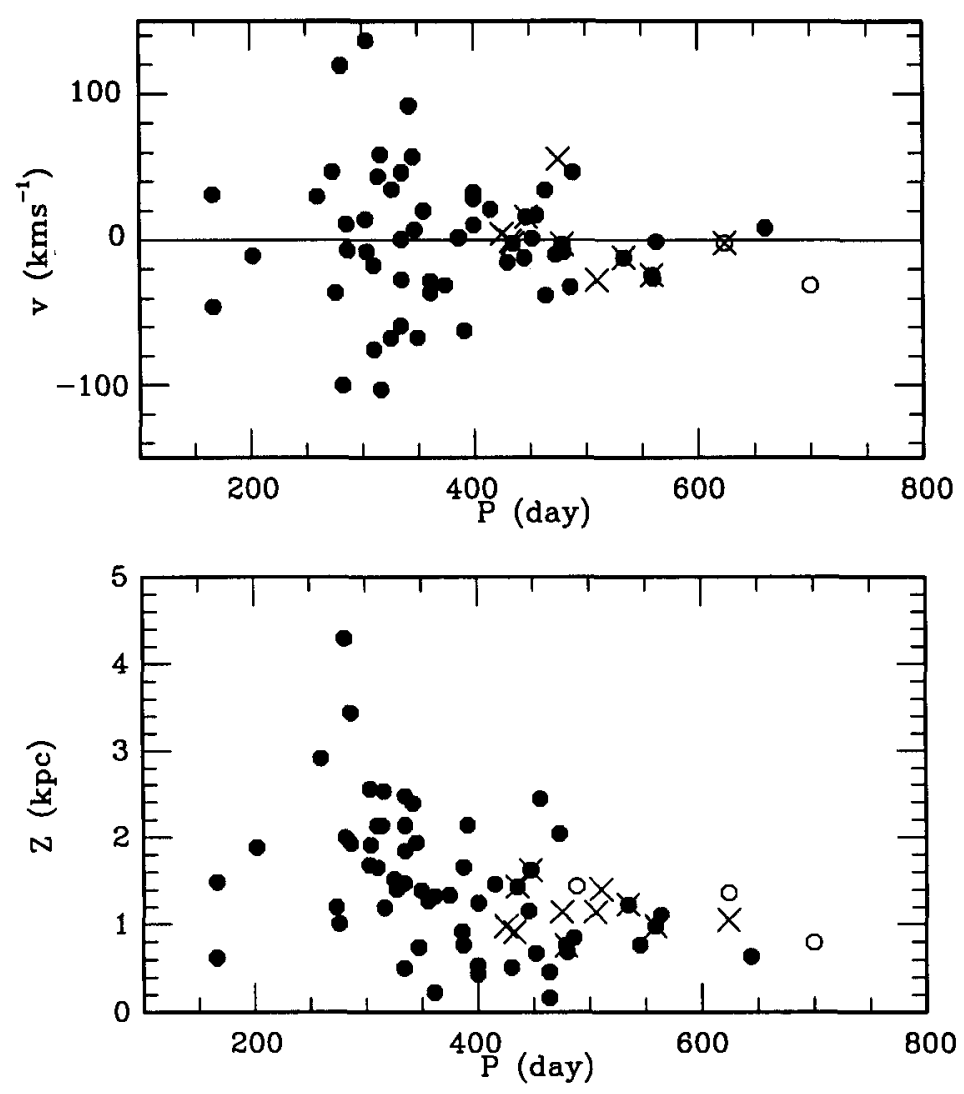

Figure 1. A comparison of the height above the Galactic Plane (Z) and the velocity $(\mathrm{v})$ as a function of pulsation period $(\mathrm{P})$ for the stars from Whitelock et al. (1994) (closed circles: M stars; open circles: Cstars) and the stars from Jura (1994) (crosses).

of the Galactic Caps for Miras of both the dusty and ordinary variety, followed by detailed analysis of their kinematics, periods and luminosities. It is worth noting that work on Miras in the Sgr I window of the Bulge by Glass et al. (1995) does not support the notion that those with thicker dust shells are less luminous than thin shelled sources of the same period. Possibly we must await follow-up studies after the DENIS and 2MASS surveys for a solution.

Note that the kinematic results on the Miras do not suggest a discrete thick and old disk, but that a continuum exists from the old disk to the thick disk and maybe beyond.

\section{The Galactic Centre}

The Galactic Centre is a very interesting region for many reasons. In the current context it is notable that it contains many $\mathrm{OH} / \mathrm{IR}$ stars to which we have an 
independent, if uncertain, distance estimate. Lindqvist et al. (1992) discovered $134 \mathrm{OH} / \mathrm{IR}$ sources within $100 \mathrm{pc}$ of the centre and van Langevelde (1992) and Jones et al. (1994) have measured luminosities for a few of them (see $\$ 2$ ). They seem to be rather different from the Miras in the Bulge in that they include stars with periods up to 1000 day. This is presumably indicative of rather more massive stars than are found in the Bulge. This may be because the population is younger or it could be the result of numerous binary mergers in the dense environment around the Centre. Lindqvist et al. showed that the $\mathrm{OH}$ sources are in rapid rotation around the centre. For this reason it has been suggested that these stars may be more closely related to the disk than to the Bulge. However the existence of at least some stars on non-circular orbits is more consistent with a halo than a disk population. I understand that more work is being done on these stars by Habing and associates and that we can look forward to seeing proper motions in the future.

\section{The Galactic Bulge}

What have we learned about the structure of the Bulge from Mira-like variables? The distribution of periods of Bulge Miras is of some interest as it we have seen above that the kinematics of local Miras are a function of their period. There are in fact still rather few regions in the Bulge for which there is anything approaching a complete sample of all periods, although a large number of Miras are catalogued. Most of what we know about the period distribution comes from the Baade windows surveyed by Lloyd Evans (1976) with some additional long-period stars discovered as IRAS sources (Glass 1986). IRAS did not detect the short-period Miras at the distance of the Bulge. The period distributions were illustrated in Fig. 3 of Whitelock (1993). Again the long period tail may be the result of binary mergers. There is a somewhat larger fraction of long-period stars in the inner Bulge than in the outer Bulge. There seem to be significant numbers of the stars which locally we would associate with the thick disk and slightly smaller numbers of those we would associate with the old disk, and everything in between. There is however no evidence, as yet, of a kinematic dependence on period among the Bulge Miras.

Miras have played a key role in the recent discovery of the bar structure associated with the Bulge. Nakada et al. (1991) looked at the distribution of colour-selected IRAS sources (most of which we have good reason to believe will be Miras) and found a longitudinal asymmetry which they interpreted as a tilt in the Bulge. Whitelock \& Catchpole (1992) looked at IRAS Miras for which we had periods and luminosities and found a tilt in the Bulge. This has been confirmed in a recent analysis of the data from the DIRBE experiment on the COBE satellite (Dwek et al. 1995).

Whitelock \& Catchpole (1992) examined the distances to IRAS Miras in two strips across the Bulge. The distances were determined from the PL relation. They found that the stars at positive longitudes are closer than those at negative longitudes, and were able to model this with an ellipsoidal Bulge tilted to the line of site by about $45^{\circ}$. This approach, using distances to individual stars, is time consuming but enormously powerful in determining detailed structure 
compared to examining the overall light distribution at any given wavelength. The interpretation of the latter requires detailed models of the numbers and types of stars in the Bulge.

Menzies (1990) obtained optical spectra of the brightest of these Miras and demonstrated that at these latitudes the Bulge is rotating at $70 \mathrm{kms}^{-1} \mathrm{kpc}^{-1}$. A few of the Bulge IRAS sources were also detected in $\mathrm{OH}$ as part of the Parkes survey of colour-selected IRAS sources by te Lintel Hekkert (1990) although most of the Bulge sources do not have sufficiently thick dust shells to make them detectable $\mathrm{OH}$ emitters at the distance of the Bulge. A far larger number of Bulge sources have been detected as $\mathrm{SiO}$ Masers from the Nobeyama radio observatory by Izumiura et al. (1994). Initially this group examined the IRAS sources in the $7^{\circ}<|b|<8^{\circ}$ strip that Whitelock et al. (1991) had demonstrated were Miras. This was so successful that they decided to extend the survey over a much larger area of the Bulge, viz. $3^{\circ}<|b|<15^{\circ}$ and $l= \pm 15$ (Izumiura et al. 1995). They measure a Bulge rotation of $75 \mathrm{kms}^{-1} \mathrm{kpc}^{-1}$, with no evidence of departure from cylindrical rotation, although the velocity dispersion is high $\left(76 \mathrm{kms}^{-1}\right)$ and the number of sources in a given latitude strip not large. (The $\mathrm{OH}$ sources near the Galactic centre are rotating much more rapidly at 1200 $\mathrm{kms}^{-1} \mathrm{kpc}^{-1}$ ). There are some tantalising asymmetries in the rotation curve and in particular an offset from zero of $18 \pm 8 \mathrm{kms}^{-1}$. This may indicate that the local standard of rest is moving towards the Galactic Centre or that there are streaming motions of the stars in the bar.

\section{Future prospects}

Finally, a few remarks about the future: We have the potential to investigate the detailed disposition and motions of old stars over a large fraction of the Galaxy; we are no longer limited to a tiny volume around the sun. Using radio observations of molecular Masers we have the ability to measure the velocities and proper motions of variables at least as far as the Galactic Centre, independent of intervening extinction. Using the results from HIPPARCOS for optical Miras and phase-lag distances to OH/IR stars we can test, calibrate and refine the distance scale. ISO observations of stars in the Magellanic Clouds will aid our understanding of the long-period variables. The DENIS and 2MASS surveys will reveal red variables throughout a large fraction of the galaxy. To make proper use of these data we will have to determine pulsation periods, and this will be time consuming. Accurate periods are a by-product of the searches for gravitational lenses in the Bulge and in the LMC. Photographic work in progress with the Anglo-Australian Schmidt will also provide periods. In the very crowded and obscured regions multiple observations with infra-red arrays will be required, and such measurements are becoming easier to make. The observations which form the basis of our understanding of the Galactic disposition of old populations are largely of stars from the extended solar neighbourhood. It will be fascinating to see how they stand up to a test on the grand scale, and this we should be able to do in the not too distant future.

Acknowledgments. I would like to thank Michael Feast and John Menzies for helpful discussions. 


\section{References}

Baud, B. et al. 1981, A\&A, 95, 156

Carney, B. W. 1993, in: Majewski S. R. (ed), Galaxy Evolution: The Milky Way Perspective, ASP Conf. Ser. 49, p. 83

Chapman, J. 1995, these proceedings

Dwek, E. et al. 1995, preprint

Feast, M. W. 1988, in: van den Bergh, S. \& Pritchet, C. J. (eds) The Extragalactic Distance Scale, ASP Conf. Ser. 4, p. 9

Feast, M. W., Woolley, R., \& Yilmaz, N. 1972, MNRAS, 158, 23

Feast, M. W. et al. 1989, MNRAS, 241, 375

Freeman, K. C., 1987, ARA\&A, 25, 603

Glass, I. S. 1986, MNRAS, 221, 879

Glass, I. S. et al. 1995, MNRAS, in press

Habing, H. J. 1988, A\&A, 200, 40

Izumiura, S. et al. 1994, ApJ, 437, 419

Izumiura, S. et al. 1995, submitted to ApJS

Jura, M. 1994, ApJ, 422, 102

Jura, M., \& Kleinmann, S. G. 1989, ApJ, 341, 359

Jura, M., \& Kleinmann, S. G. 1992, ApJS, 79, 105

Jura, M., Yamamoto, A., \& Kleinmann, S. G. 1993, ApJ, 413, 298

Jones, T. J. et al. 1994, AJ, 107, 1111

Lindqvist, M., et al. 1992, A\&AS, 92, 43

Lloyd Evans, T. 1976, MNRAS, 174, 169

Majewski, S. R. 1993, ARA\&A, 31, 575

Menzies, J. W. 1990, in: Jarvis B. J. \& Terndrup D. M. (eds), Bulges of Galaxies, ESO/CTIO Workshop, p. 115

Nakada, Y. et al. 1991, Nature, 353, 140

Ojha, D. K. et al. 1994, A\&A, 290, 771

Sivagnanam, P. et al. 1989, A\&A, 211, 341

te Lintel Hekkert, P. 1990, thesis, Leiden

van Langevelde, H. J. 1992, thesis, Leiden

Whitelock, P. A. 1990, in: Cacciari, C. \& Clementini, G. (eds), Confrontation between Stellar Pulsation and Evolution, ASP Conf. Ser., 11, p. 365

Whitelock, P. A. 1993, in: Dejonghe, H. \& Habing, H. J. (eds), Galactic Bulges, IAU Sym 153, Kluwer, p. 39

Whitelock, P. A., \& Catchpole, R. M. 1992, in: Blitz L. (ed), The Center, Bulge and Disk of the Milky Way, Kluwer, p. 103

Whitelock, P. A. et al. 1994, MNRAS, 267, 711

Whitelock, P. A. et al. 1995, submitted to MNRAS

Wood, P. R. et al. 1992, ApJ, 397, 552 


\section{Discussion}

Stift: You find no metallicity dependence of the PL relation. How does the assumed LMC distance modulus affect this conclusion?

Whitelock: We find the distance of the Miras in the LMC and the globular clusters with respect to the RR Lyrae stars. So if you change the distance to the Clouds you will change the distance to the clusters in the same way. The magnitudes of the Miras with spectroscopic and trigonometric parallaxes are not tied in the same way to the LMC.

Bedding: For how many Miras will HIPPARCOS give reasonable distances?

Whitelock: Probably about half a dozen or so. It depends on the final accuracy of the HIPPARCOS positions (Catherine Turon is nodding!).

Chapman: From present observations of stellar periods against other quantities, it looks very unlikely that the P-L relationships will be found to be valid for $\mathrm{P}$ $\gtrsim 800$ days. I interpret this in the sense that the most massive stars $\left(\gtrsim 4 M_{\odot}\right)$ do show strong evolution in $\mathrm{P}$ - so that a star of given mass and luminosity could have a large range of periods between, say, 800 and 2000 days.

Habing: With respect to the question how much the period of a given star can increase during these late phases of evolution: the Feast (1963) paper showed clearly that there is a relation between period and kinematics - how much freedom does this paper leave one to speculate about period increases?

Whitelock: I think all we can say is that there is no observational evidence for changes in $\log (P)$ of more than about 0.05 . But we obviously cannot rule out the possibility that much larger changes occur very rapidly, so that we do not actually observe stars in that state.

Shibahashi: I was very much impressed by using the beautiful P-L relationship that you showed us. However, I also got complaint by such a simple relation between the periods and the luminosities of Mira variables. The periods of a star are mainly governed by two parameters, its mass and radius. Could you say some words to explain the unique P-L relation of Mira variables by which the period is related with only one parameter, $\mathrm{L}$ ?

Whitelock: It is a very curious situation. What we seem to be finding is that there is a particular mass/age/metallicity relationship which is the same in various parts of the Galaxy and in the Magellanic Clouds. 